\title{
Absence of High Penetrance Genes Mutations in Familial Breast Cancer in Mizo-Mongloid Population, Northeast India
}

\section{Doris Zodinpuii}

Mizoram University Department of Biotechnology

\section{Bawitlung Zothankima}

Mizoram State Cancer Institute

Jeremy Lalrinsanga Pautu

Mizoram State Cancer Institute

Doris Lallawmzuali

Mizoram State Cancer Institute

\section{Ashok Kumar Varma}

Advanced Centre for Treatment Research and Education in Cancer

\section{Ashok kumar Varma}

Homi Bhabha National Institute

\section{Rajendra Bose Muthukumaran}

Mizoram University

Nachimuthu Senthil Kumar ( $\nabla$ nskmzu@gmail.com )

Mizoram University https://orcid.org/0000-0002-0192-2133

\section{Brief report}

Keywords: BRCA1, TP53, PTEN, CDH1, CHEK2, XRCC2, Familial Breast Cancer, Polymorphisms

Posted Date: April 9th, 2020

DOI: https://doi.org/10.21203/rs.3.rs-21839/v1

License: (c) (1) This work is licensed under a Creative Commons Attribution 4.0 International License. Read Full License 


\section{Absence of High Penetrance Genes Mutations in Familial Breast Cancer in Mizo- Mongloid Population, Northeast India}

Doris Zodinpuii ${ }^{1}$, Bawitlung Zothankima ${ }^{2}$, Jeremy Lalrinsanga Pautu ${ }^{3}$, Doris Lallawmzuali $^{4}$, Ashok Kumar Varma ${ }^{5,6}$, Rajendra Bose Muthukumaran ${ }^{7}$, Nachimuthu Senthil Kumar ${ }^{1}$

${ }^{1}$ Department of Biotechnology, Mizoram University, Tanhril, Mizoram, India

${ }^{2}$ Department of Radiation Oncology, Mizoram State Cancer Institute, Zemabawk, Aizawl, Mizoram, India

${ }^{3}$ Department of Oncology, Mizoram State Cancer Institute, Zemabawk, Aizawl, Mizoram, India

${ }^{4}$ Department of Pathology, Mizoram State Cancer Institute, Zemabawk, Aizawl, Mizoram, India

${ }^{5}$ Tata Memorial Centre, Advanced Centre for Treatment, Research and Education in Cancer, Kharghar, Navi Mumbai, India

${ }^{6}$ Homi Bhabha National Institute, Anushaktinagar, Mumbai, India

${ }^{7}$ Department of Chemistry, Mizoram University, Tanhril, Mizoram, India

Correspondence: Nachimuthu Senthil Kumar

Department of Biotechnology, Mizoram University, Tanhril, Mizoram, India, Pin796009, Tel: +91- 9436352574, E-mail: nskmzu@gmail.com

Orcid no: 0000-0002-0192-2133 


\begin{abstract}
Abstarct
Background Breast cancer is the most prevalent cancer and leading cause of death among women globally. The present study focuses on screening germline mutations of breast cancer susceptibility genes among the unexplored Mizo breast cancers of culturally and historically homogenous population, living a unique life style habits in terms of diet and tobacco usage.
\end{abstract}

Methods Mutation screening was performed using Sanger sequencing in complete coding region of BRCA1 and frequently mutated exons of TP53, PTEN, CDH1, CHEK2 and XRCC2. Several online mutation prediction tools and databases were used to check the pathogenicity of the polymorphisms observed.

Results: We observed eight polymorphisms in total, in which, one variants p.P1544P in BRCA1 gene was found to be novel. No variants were found to have a potential impact on protein since all the polymorphisms are of silent substitutions. No genetic alteration was observed in the studied exons of each of TP53, PTEN, CDH1, CHEK2 and XRCC2.

Conclusion: To our knowledge, the present study focusing on familial breast cancer is the first time to analyzed the prevalence of breast cancer susceptibility gene mutations using direct sequencing in Mizo population. Even though, we do not find significant amino acid change, our results suggest the need for further evaluation of broader panel genes and a challenge to screen larger sample size to establish the contribution of these gene mutations in this population.

Keywords: BRCA1, TP53, PTEN, CDH1, CHEK2, XRCC2, Familial Breast Cancer, Polymorphisms. 


\section{Introduction:}

Breast cancer is a complex multifactorial disease regulated by several factors. Globally, breast cancer is the top most prevalent cancer and leading cause of mortality among women [1,2]. In 2018, breast and lung cancer holds the same highest position $(11.6 \%)$ when both the sexes are considered. And when only females were considered, the number of new cases $(24.2 \%)$, the estimated incidence and mortality rate is also highest for breast cancer [3].

In the North Eastern part of India, the prevalence of breast cancer is considerably higher then the rest of India probably due to the genotoxic stress from tobacco exposure [4]. According to the latest report of National Cancer Registry Program (NCRP), breast cancer rank the third highest cancer $(13.0 \%)$ among the Mizo women populace [5]. Hence, the rapid rise in the number of incidence and mortality due of breast cancer among tribal women in rural areas like Mizoram over the past few year calls for an extensive study and exploration on this area of cancer.

The main causal factor for developing breast cancer is germline mutations in breast cancer susceptibility genes [6]. Germline mutations in BRCA1 gene account for $5-10 \%$ of breast cancers in white Europeans and carriers of this gene mutation have $85 \%$ lifetime risk of developing breast cancer by age 70 years [7]. The spectrum of BRCA1 gene mutations has been screened among different population, especially in western populations and majority of these have involved hereditary breast and ovarian cancer families [8].

Other genes conferring breast cancer susceptibility, albeit with lower frequency and penetrance include TP53, PTEN, CDH1 and CHEK2 [6,7]. 
Furthermore, in exome sequencing of familial breast cancer patients, deleterious XRCC2 germline mutation has been identified $[9,10]$. Clinical significance of breast cancer susceptibility genes and their associated mutations relies on their prevalence in a specific population [11]. However, such studies in Mizo population remain unexplored.

This is the first report that focuses on the contributory role of breast cancer susceptibility gene mutation among this population. This population has a very high risk of breast cancer and unique life style habits in terms of diet and tobacco usage as well as is homogenous, both culturally and historically $[12,13]$. Hence, it is interesting to screen the frequently mutated genes for breast cancer and test whether the gene mutations are prevalent and are pathogenic in nature.

\section{Materials and Methods:}

\section{Sample collection, DNA isolation and gene amplification}

For this study, 50 unrelated local ethnic breast cancer patients having self reported family history of breast and ovarian cancers and 50 healthy controls having no history of any type of cancers were considered. Blood samples were drawn with the consent of the participants admitted in Mizoram State Cancer Institute (MSCI), Aizawl, Mizoram. Isolation of genomic DNA was done using QIAamp DNA mini kit (catalogue: 51306) from $200 \mu 1$ blood according to the manufacturers protocol. The present work has been approved by the ethical committees of Civil Hospital, Aizawl (B.12018/1/13-CH(A)/IEC/33 dtd.15/10/2014) and Institutional Human Ethical Committee, Mizoram University. 
Gene amplification was carried out using self-designed specific primers for the specified region of interest (Supplementary Table 1). Polymerase chain reaction (PCR) was performed using $25 \mu 1$ total reaction volumes, each containing template DNA (100 ng/ $\mu \mathrm{l})$, forward and reverse primers (10 pM/ $\mu \mathrm{l}), 10 \mathrm{X}$ PCR buffer $(2.5 \mu \mathrm{L})$, dNTPs $(10 \mathrm{mM})$, Taq polymerase (1U) and milli-q $\mathrm{H}_{2} \mathrm{O}$ (to make up the volume). PCR conditions used were as follows, initial denaturation at $95^{\circ} \mathrm{C}$ for 5 minutes, denaturation at $94^{\circ} \mathrm{C}$ for 30 seconds, annealing for $50-63^{\circ} \mathrm{C}$ for 35 seconds, extension at $72^{\circ} \mathrm{C}$ for 35 seconds and final extension at $72^{\circ} \mathrm{C}$ for 3 minutes. The PCR amplified products were loaded in $1.2 \%$ agarose gel and the success of amplification was checked using Bio-rad Gel documentation system.

\section{Purification, Sequencing and Sequence analysis}

The PCR amplified products were purified to remove excess salts, primers and dNTP's using Qiagen purification kit and Exonuclease and shrimp Alkaline phosphatase (Exo-sap) using the standard manufacturers protocol. Applied Biosystem, 3500 Genetic Analyzer was used to sequence the purified products from both the direction using forward and reverse primers for reading accuracy. Sequence chromatograms obtained was analyzed using FinchTV version 1.4.0 and was aligned using NCBI BLASTN [14]. Ensembl and HUGO Gene Nomenclature Committee databases were used to check correct exon amplification $[15,16]$. Mutation Taster, Breast Cancer Information Core (BIC) BRCA, ARUP BRCA, PolyPhen- 2, Align GVGD and ClinVar databases were used to analyze the pathogenicity and novelty of the observed polymorphisms $[17,18,19,20,21,22]$. 


\section{Results:}

Isolated genomic DNA and PCR amplified products of the selected exons were given in supplementary figures 1 . From three exons $(11,13$ and 15) of BRCA1 gene, eight polymorphisms were observed, in which four are synonymous and other four are non-synonymous. Among them g.95900A $>$ T: c.4772A $>$ T: p.P1544P from exon 15 of BRCA1 gene was found to be novel. The remaining 7 polymorphisms of BRCA1 gene have been reported by several genetics clinics and research groups for other populations in databases such as BIC BRCA, ARUP BRCA, dbSNP and ClinVar. We do not observe any significant amino acid changed in BRCA1 gene since all the polymorphisms are of silent substitutions. Chromatographic file for novel and other polymorphisms were shown in figure 1. Details of polymorphisms and their predicted scores are given in table 1. No genetic alteration was observed in the studied exons of each of TP53, PTEN, CDH1, CHEK2 and XRCC2.

\section{Discussion:}

From the analysis, eight variations were observed in total, out of which two variants was found to be novel. Among the studied samples, no genetic alteration was identified in TP53, PTEN, CDH1, CHEK2 and XRCC2 genes. In BRCA1 gene, no significant amino acid changes were observed since the polymorphisms are either synonymous or silent substitution. Except for the novel polymorphism, the remaining polymorphisms have been repeatedly reported to have no clinical significance in BIC BRCA and ClinVar database by several genetics clinics from all over the world and majority of these polymorphisms reported in these repositories are unpublished [7].

p.L771L, p.S694S, p.P871L, p.E1038G, p.K1183R from exon 11 and p.S1436S from exon 13 of BRCA1 gene has been reported by many studies as 
polymorphisms or variants of uncertain significance with minor allele frequency (MAF) more than 25\% [23,24,25]. p.S694S was reported to be associated with age at diagnosis [26]. Most of the observed polymorphisms are inherited as part of shared haplotype and are in significant linkage disequilibrium (LD). p.L771L, p.S1436S, p.P871L, p.E1038G and p.K1183R has also been reported for other Indian population and among them, p.P871L, p.E1038G and p.K1183R were listed on top 20 BIC entries. There are reports that absence of BRCA1 p.K1183R increases breast cancer risk suggesting the protective effect of the polymorphism [7,24]. The possible hypothesis behind cancer occurrence despite the presence of p.K1183R polymorphism in our studied cases could be the balance between DNA damage and repair that determines the individual susceptibility to breast cancer. DNA damages caused by certain environmental factors such as those of chemicals and radiations that are not repaired fully could wake the genomes stability leading to carcinogenesis [27].

A meta-analysis of 24 studies consisting of 13762 cases and 22388 controls has found no association between BRCA1 p.P871L and risk of breast cancer. Despite the fact that this polymorphism leads to substitution of amino acid proline to leucine at position 871 , which is part of interaction region for another critical homologous recombination protein called RAD51 and has been considered to confer cancer susceptibility, they observed a significant association with decreased risk of overall cancer in either homozygous or heterozygous condition [27].

Since there has been an inconsistent results for the association of $\mathrm{p} . \mathrm{E} 1038 \mathrm{G}$ with risk of sporadic breast cancer, Ricks-Santi et al. (2013), performed genotyping of the variant using 1005 cases and 1765 controls and found no evidence of independent association between the genotypes of p.E1038G and risk of breast cancer either in pre 
or postmenopausal women. However, among premenopausal breast cancer with carriers of p.E1038G polymorphism, an interaction was observed between genotype and their smoking status, specifically, smokers with TT genotype were shown to be at a higher risk.

Two polymorphism (p.P1544P and p.D1546Y) were observed in exon 15 of BRCA1 gene in which p.P1544P, a synonymous mutation was found to be novel since it has not been reported in any known breast cancer databases or any other existing databases. Interestingly, p.D1546Y has been found reported in databases only for European (Non Finnish) population with a very low MAF (MAF $>0.00003295)$ and yet by only genetics clinics in BIC BRCA, ClinVar and ExAC databases [18,22,28].

There were no polymorphisms observed in TP53, PTEN, CDH1, CHEK2 and $\mathrm{XRCC} 2$. Since it is well established that breast cancer is a regulated by both genetics and epigenetic factors and the underlying genetic mechanisms vary from different population [2,29], it is possible that we do not observed mutations associated with breast cancer, despite, the fact that exon selections are based on the maximum number of codons on which breast cancer related mutations have been reported. Our study provides significant preliminary information, suggesting the need to screen larger panel genes using high throughput sequencing technology, which could further provide gene mutations prevalent in the Mizo population.

\section{Conclusion:}

The current study calls for an extensive exploration of broader breast cancer panel genes other than BRCA1 gene using high throughput sequencing technology to establish the frequency, penetrance and significance on their contribution towards breast cancer progression in this unexplored tribal population of North-east India, 
since it could serve as a significant data for genetic counseling. Moreover, identification of individuals with pathogenic mutations is clinically relevant since several strategies are available for risk reduction, early detection, and better management of Breast cancer. Also, from this study, it is evident that whole exome sequencing would be a better approached to investigate other unexplored population like Mizoram, where there is no preliminary data. To our knowledge, the present study is the first time to analyzed the entire coding regions of BRCA1 and other exons of breast cancer susceptibility gene using direct sequencing in Mizo population.

\section{Ethics approval and consent to participate}

The present work has been approved by the ethical committees of Civil Hospital, Aizawl (B.12018/1/13-CH(A)/IEC/33 dtd.15/10/2014) and Institutional Human Ethical Committee, Mizoram University.

\section{Consent for publication}

Not applicable

\section{Availability of data and materials}

The datasets generated during and analyzed during the current study are available from the corresponding author on reasonable request.

\section{Competing interests}

The authors declare that they have no competing interests.

\section{Funding}


The authors received no financial support for the research, authorship, and/ or publication of this article.

\section{Authors' contributions}

$\mathrm{DZ}$, NSK, AKV and RBM design of the project. DZ performed the experiment and data analysis and writing of the manuscript. BZ, JLP and DL provide the Breast cancer samples. BZ, JLP, DL, AKV, RBM, NSK review the manuscript.

\section{Acknowledgement}

The authors would like to acknowledge DBT-Advanced State Level Biotech Hub and DBT-Bioinformatics Infrastructure Facility, Mizoram University, Aizawl for the completion of their work. 


\section{References}

1. Atif N, Khalid M, Chughtai O, Asif Saad, Rashid M, AS Chughtai. Role of immunohistochemical markers in breast cancer and their correlation with grade of tumour, our experience. Int Clin Pathol J. 2018;6(3):141-145.

2. Sirisena ND, Adeyemo A, Kuruppu AL, Neththikumara N, Samaranayake N, Dissanayake VHW. Genetic determinants of sporadic breast cancer in Sri Lankan women. BMC Cancer. 2018;18:180.

3. Globocan. International Agency for Research on Cancer, 2018. http:/gco.iarc.fr/today/data/factsheets/cancers/20-Breast-fact-sheet.pdf .Accessed 6 August 2019.

4. Saxena S, Kaushal M, Sharma J, Zomawia E, Kapur S. Genomic alteraions in breast cancer patients from Northeast India using 10K SNP arrays. Genome Biology. 2010;11:34.

5. Population $\quad$ Base $\quad$ Cancer 2014. http://www.ncdirindia.org/NCRP/ALL_NCRP_REPORTS/PBCR_REPORT_201 2_2014/ALL_CONTENT/PDF_Printed_Version/Chapter2_Printed.pdf. Accessed 6 August 2019.

6. Mahdavi M, Nassiri M, Kooshyar MM, Vakili-Azghandi M, Avan A, Sandry R, et al. Hereditary breast cancer; Genetic penetrance and current status with BRCA, J Cell Physiol. 2019;234:5741-5750.

7. Sharma-Oates A, Shaaban AM, Tomlinson I, Wynne L, Cazier J-B, Sundar S. Heterogeneity of germline variants in high risk breast and ovarian cancer susceptibility genes in India. Precis Clin Med. 2018;1(2):75-87.

8. Akilzhanova AR, Nyshanbekkyzy B, Nurkina ZM, Shtephanov II, Makishev AK, Adylkhanov TA, et al. BRCA1 and BRCA2 Gene Mutations Screening In 
Sporadic Breast Cancer Patients In Kazakhstan. Cent Asian J Glob Health. $2013 ; 2: 1$.

9. Park DJ, Lesueur F, Nguyen-Dumont T, Pertesi M, Odefrey F, Hammet F, et al. Rare Mutations in XRCC2 Increase the Risk of Breast Cancer. Am J Hum Genet. 2012;90:734-739.

10. Hilbers FS, Wijnen JT, Hoogerbrugge N, Oosterwijk JC, Collee MJ, Peterlongo $\mathrm{P}$, et al. Rare variants in XRCC2 as breast cancer susceptibility alleles. J Med Genet. 2012;49(10):618-20.

11. Muhammad N, Nawaz R, Khan FA, Naeemi H, Rashid MU. Inherited genetic susceptibility to breast cancer in Pakistan. J Cancer Allied Spec. 2018;4(2):2.

12. India's Cancer Capital, Mizoram, Cancer India. http://cancerindia.org.in/mizoram-indias-cancer-capital/. Accessed 9 August 2019

13. Mizoram Has Highest Number of Inter-Caste Marriages in India With 55\%, The. Wire https://thewire.in/uncategorised/mizoram-has-highest-number-of-intercaste-marriages-in-india-with-55. Accessed 2 August 2019.

14. National Centre for Biotechnology Information. www.ncbi.nlm.nih.gov/blast. Accessed 29 August 2019.

15. Ensembl. https://asia.ensembl.org/index.html. Accessed 29 August 2019.

16. HUGO Gene Nomenclature Committee. https://www.genenames.org. Accessed 30 August 2019.

17. Mutation Taster. http://www.mutationtaster.org. Accessed 30 August 2019.

18. Breast Cancer Information Core (BIC) BRCA. https://research.nhgri.nih.gov/bic/. Accessed 2 August 2019.

19. ARUP BRCA. http://arup.utah.edu/database/BRCA/Home/BRCA1_landing.php. 
Accessed 15 August 2019.

20. PolyPhen- 2. http://genetics.bwh.harvard.edu/pph2/. Accessed 3 September 2019.

21. Align GVGD. http://agvgd.hci.utah.edu. Accessed 3 September 2019.

22. ClinVar. https://www.ncbi.nlm.nih.gov/clinvar/ . Accessed 3 September 2019.

23. Borg A, Haile RW, Malone KE, Capanu M, Diep A, Törngren T, et al. Characterization of $B R C A 1$ and $B R C A 2$ Deleterious Mutations and Variants of Unknown Clinical Significance in Unilateral and Bilateral Breast Cancer: The WECARE Study. Hum Mutat. 2010; 31(3):E1200-E1240.

24. Buleje J, Guevara-Fujita M, Acosta O, Huaman FDP, Danos P, Murillo A, et al. Mutational analysis of BRCA1 and BRCA2 genes in Peruvian families with hereditary breast and ovarian cancer. Mol Genet Genom Med. 2017;5(5): 481494.

25. Hondow HL, Fox SB, Mitchell G, Scott RJ, Beshay V, Wong SQ, et al. A highthroughput protocol for mutation scanning of the BRCA1 and BRCA2 genes. BMC Cancer. 2011;11:265.

26. Ricks-Santi L, McDonald JT, Gold B, Dean M, Thompson N, Abbas M, et al. Next generation sequencing reveals high prevalence of Brca1 and Brca2 variants of unknown significance in early-onset breast cancer in African American Women. Ethnicity \& Disease. 2017;27:2.

27. Miao L, Yu Y, Ji Y, Zhang B, Yuan Z, Du Y, et al. Association between BRCA1 P871L polymorphism and cancer risk: evidence from a meta-analysis. Oncotarget. 2017;8(18): 30587-30594.

28. ExAC. http://exac.broadinstitute.org/variant/17-41226387-C-A. Accessed 4 September 2019.

29. Adejumo P, Aniagwu T, Oluwatosin A, Fagbenle O, Ajayi O, Ogungbade D, et 
al. Knowledge of Genetic Counseling Among Patients With Breast Cancer and Their Relatives at a Nigerian Teaching Hospital. Journal of Global Oncology. 2018; doi.org/10.1200/JGO.17.00158. 


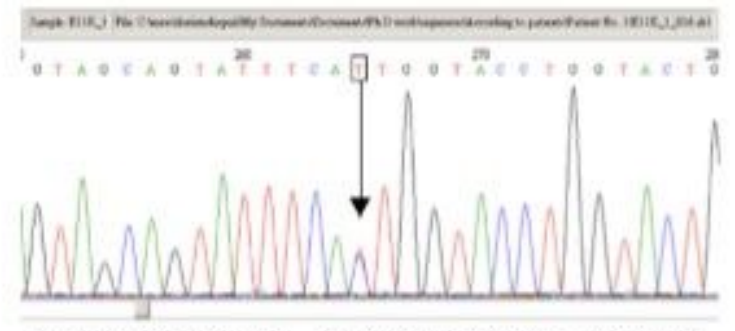

A: BRCA1 Exon -11 g. $77054 \mathrm{~T}>$ C p.L771L

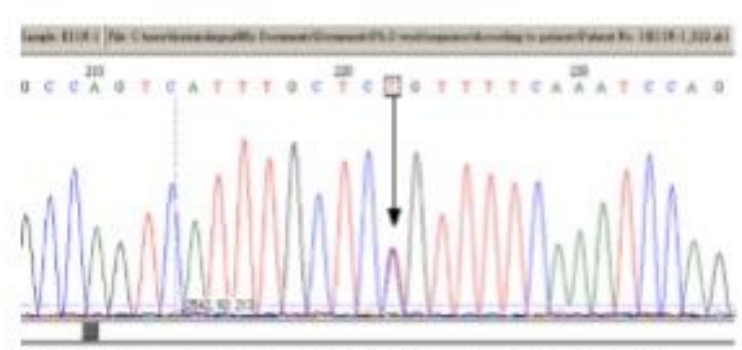

C: BRCA1 Exon-11 g.77355C $>$ T p.P871L

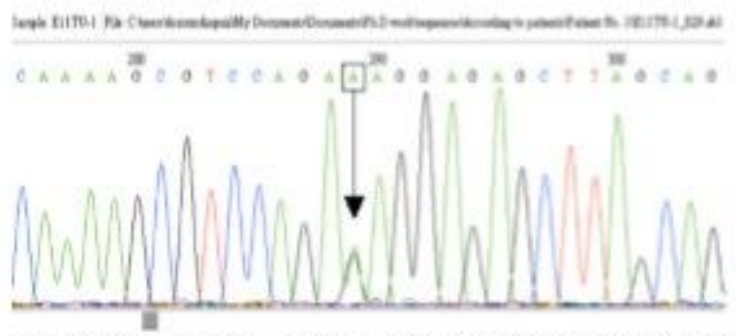

E: BRCA1 Exon-11 g.78291A>G p.K1183R

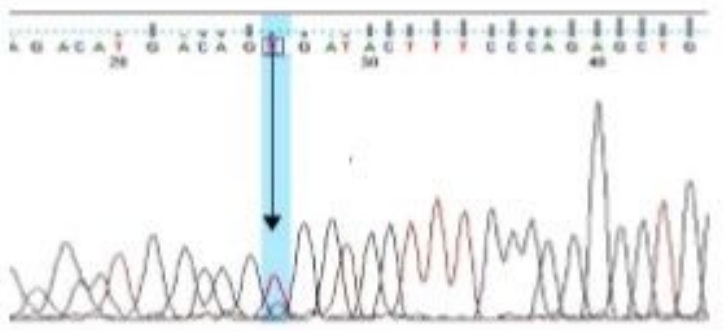

B: BRCA1 Exon 11 g.76825C>T p.S694S

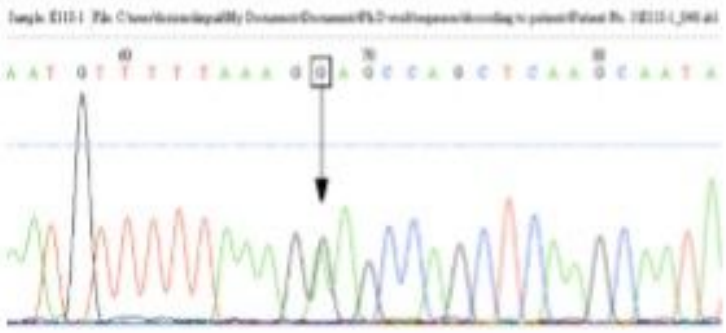

D: BRCA1 Ex-11: g.77856A>G: p.E1038G

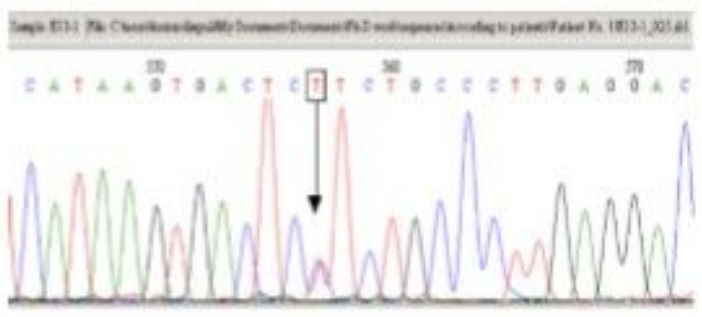

F: BRCA1 Exon-13 g.87821T>C p.S1436S

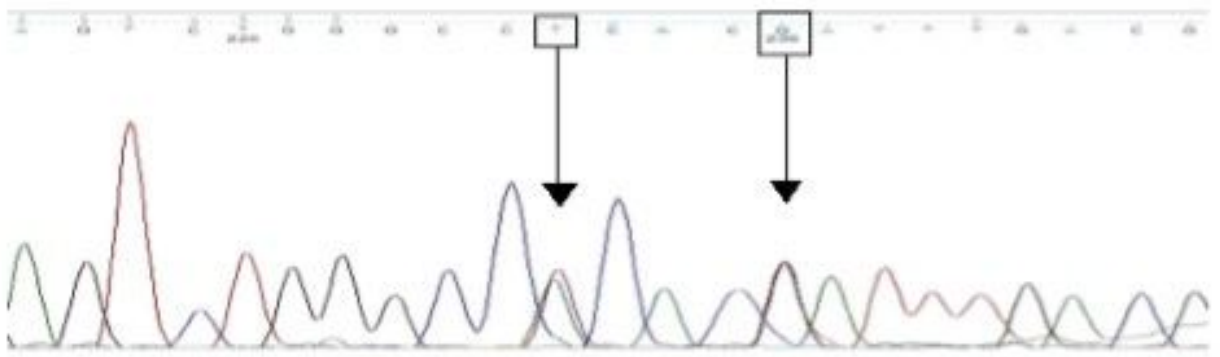

G: BRCA1 Exon-15 (i) g.95900A>T p.P1544P (ii) g.95904G>T p.D1546Y respectively.

\section{Figure 1}

Chromatogram showing observed polymorphism in BRCA1 gene, showing novel polymorphisms p.P1544P in exon 15. 


\section{Supplementary Files}

This is a list of supplementary files associated with this preprint. Click to download.

- Table..pdf

- supplementarymaterials..pdf 